\title{
Solving the Micro to Macro Problem: A new application for milli X-ray fluorescence $\mathrm{X}$-ray spectrum imaging
}

\author{
Jeffrey M. Davis*, Dale E. Newbury ${ }^{*}$, Nicholas W.M. Ritchie* and Edward P. Vicenzi** \\ * National Institute of Standards and Technology, Gaithersburg, MD 20899 \\ ** Smithsonian Institution, Museum Conservation Institute, Suitland, MD 20746
}

X-ray fluorescence (XRF) has been a mainstay of analytical chemistry for over 50 years. XRF has a number of analytical advantages over electron excited X-ray microanalysis including lower detection limits for high atomic number elements, the ability to work in an atmospheric pressure environment, and the ability to work with insulating samples without conductive coatings. XRF has traditionally been a bulk characterization technique, primarily because the size of the X-ray beam is often $1 \mathrm{~mm}$ or more. However, with polycapillary optics applied to focus the primary X-ray beam, it is possible to reduce the size of the X-ray beam without the significant signal loss associated with pinhole apertures [1]. The finely focused X-ray beams have nominal diameters between $10 \mu \mathrm{m}$ and $100 \mu \mathrm{m}$, and are called "milli" beams in order to distinguish them from the nanometer to micrometer sized beams routinely achieved at synchrotron sources [2]. With the finely focused "milli" beams, the range of applications for $\mathrm{mXRF}$ can be expanded to include particle analysis and the X-ray imaging of heterogeneous materials.

The development of milli X-ray fluorescence X-ray spectrum imaging (mXRF-XSI) was spurred on by the need to map areas greater than several square centimeters without excessive tiling of smaller X-ray images such as those typically collected on the electron probe or SEM. By recording a full X-ray spectrum (up to $40 \mathrm{keV}$ in many instruments) at each pixel, it is possible to collect images without losing the spectroscopic data. Further, by scanning the stage beneath a stationary probe, the scale limit for an X-ray image becomes equal to the limit of stage travel. This unique combination of stage scanning and hyperspectral imaging has three unique uses:

1. "Road mapping," defined as producing X-ray maps of a large specimen so as to identify areas for further microstructural investigation. Specifically, this technique takes advantage of the hyperspectral nature of the data to find the "needle in a haystack," even when the "needle" isn't being sought.

2. Large feature mapping takes advantage of the mid sized beam in order to map features several square centimeters in size. Figures 1 and 2 are excellent examples of mapping large features of heterogeneous materials.

3. Imaging difficult samples such as insulators, wet samples and samples not suitable for low vacuum environments.

This talk will focus on the development and application of mXRF-XSI and provide laboratory examples of the macro to micro problem.

\section{References}

1) Carpenter, D.A., and Taylor, M.A., "Fast, High Resolution X-ray Microfluorescence Imaging." Advances in X-ray Analysis; [34], 217 (1991)

2) Rindby, A et al, "Microbeam Technique for Energy-Dispersive X-ray Fluorescence." Advances in X-ray Analysis; [30], 109 (1989) 


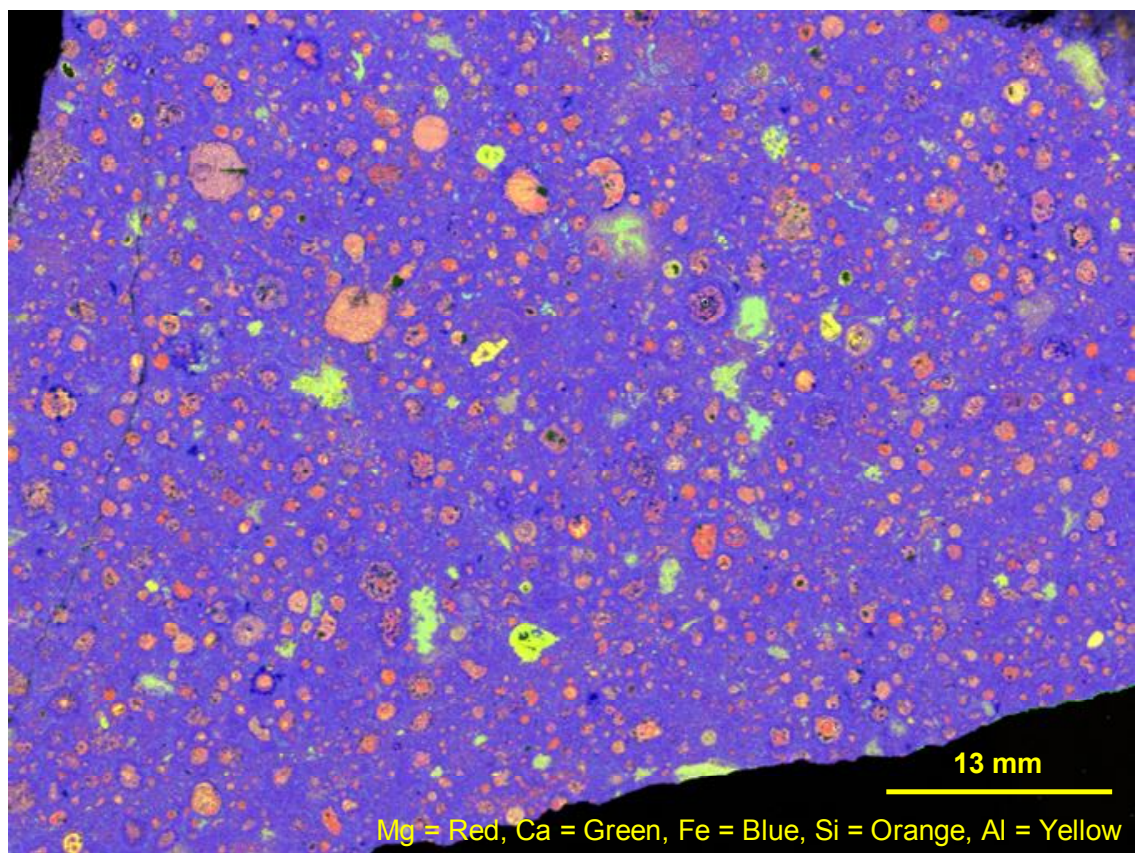

Figure 1: Large Area Map of the Allende Meteorite

A color overlay combining the X-ray images of magnesium (red), calcium (green), iron (blue), silicon (orange) and aluminum (yellow). The meteorite section is too large to produce a thin section, and has a number of interesting large scale features such as chondrules and calcium-aluminum inclusions (CAIs).

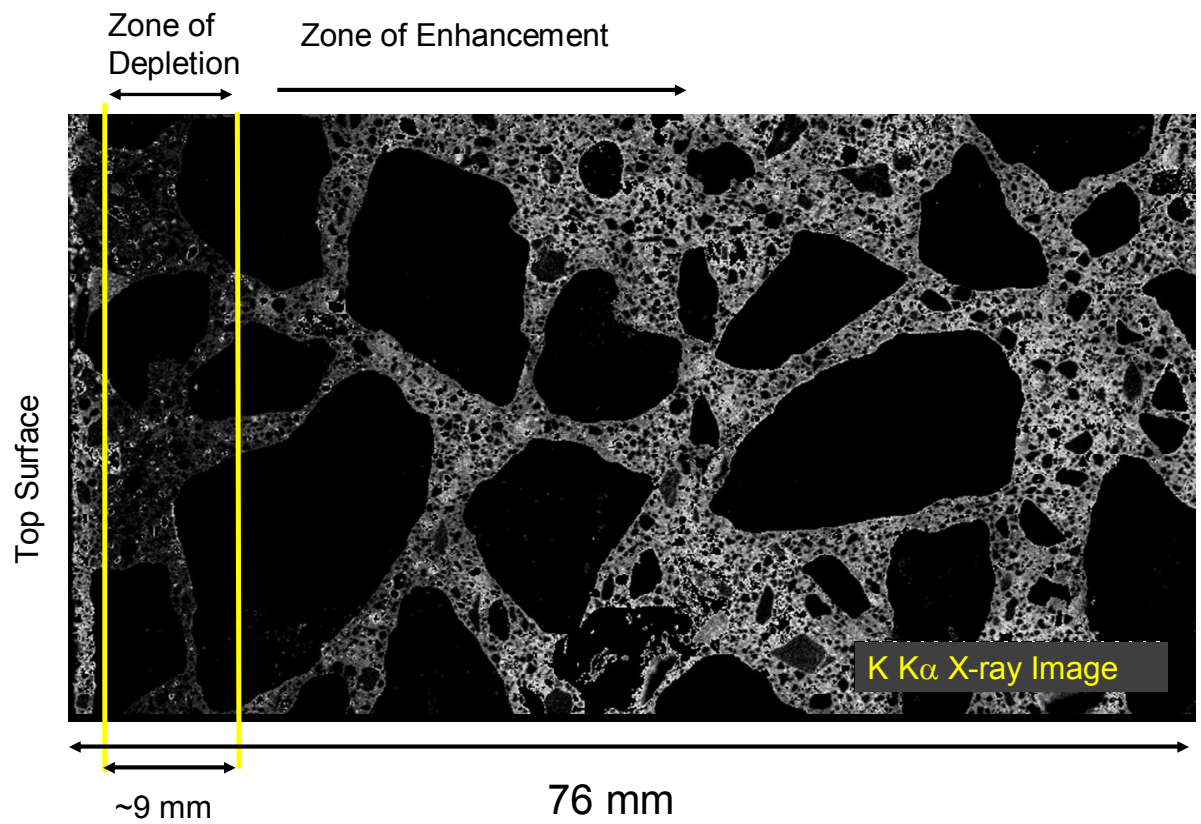

\section{Figure 2: Potassium X-ray image of a Texas Bridge Core}

This core section shows a unique potassium infiltration gradient from the driving surface (left side) to the bottom. The zone of depletion (where very little potassium is present) below the surface is part of a complex ion infiltration mechanism that is not well understood in the cement and concrete research community. 